

Lattice-gas model

The molecular dynamics analysis remains computationally very heavy despite ignoring the substrate structure. Moreover, the recent ideas about the spreading mechanisms put forward by Forcade and Mate need further investigation and clarification. For these reasons we returned to the Monte Carlo method but used an Ising or lattice-gas model that is richer from a statistical mechanics point of view [11]. The fluid particles were assumed to interact with their nearest neighbours in a cubic lattice and with the flat, unstructured substrate through the long-range potential $V(z)=-A / z^{3}$. The dynamics were assumed to obey Kawasaki spinexchange dynamics, which conserves the amount of fluid matter in the simulation system. The model therefore allows for volatility, the amount of which can be changed by adjusting the Hamaker constant $A$ and the temperature in the simulations.

As in the molecular dynamics simulations, we found dynamic layering and linear precursor growth at early times crossing over to diffusive spreading at late times. These features are shown in Fig. 3 giving snapshots of the development with time of the profile of an initially ridge-shaped droplet profile as well as the average densities of the droplet for early and late times.

\section{Tracer Studies}

We have studied the fluxes at different times of tracer particles within the fluid phase and through the vapour phase via the evaporation-condensation mechanism. At early times, when growth is linear or nearly so, the flow of matter is concentrated in the fluid interface region of the droplet cap and the flux of particles is predominantly vertically downwards towards the substrate. This results in fast precursor growth and a purely horizontal flux in the precursor. For late times, when growth is diffusive, the fluxes are purely horizontal in the precursor and predominantly horizontal in the second and third layer. Increased vertical fluxes were only observed in the neighbourhood of the layer edges. Under these conditions, growth of the precursor is dominated by particles migrating off from the second layer edge and performing random walks on the precursor layer until they find holes to fill or rough precursor edges to stick with. It is believed that these mechanisms explain the experi-

Fig. 3 - Snapshots from a Monte Carlo simulation of the development with time of a rectangular ridged-shaped initial droplet made from a three-dimensional lattice gas [11]. The droplet (thickness) profile (a, upper) as given by the droplet interface corresponding to half the maximum fluid density. Average densities of the droplet are plotted for early ( $b$, middle) and late (c, lower) times.

mental results of Forcada and Mate more satisfactorily than the evaporation-condensation process these authors invoked.

The models that have been discussed assume fluid molecules without any geometric structure. However in some silicon oils the molecules have a clear chain-like structure which implies that excluded volume and chain entanglement need to be taken into account. These aspects, which are treated fully in [12], essentially involve details beyond the scope of this brief review of the status of microscopic treatments of droplet spreading

[1] Heslot F., Fraysse N. \& Cazabat A.M. Nature (London) 338 (1989) 640; Heslot F, Cazabat A.M. \& Levison P., Phys. Rev. Lett. 62 (1989) 1286

[2] de Gennes P.G., Rev. Mod. Phys. 57 (1985)
827.

[3] Forcada M.L. \& Mate M., Nature (London) 363 (1993) 527.

[4] Joanny J.F. \& de Gennes P.G., J. Phys. (Paris) 47 (1986) 121

[5] Cazabat A.M., Contemp. Phys. 28 (1987) 347.

[6] Abraham D.B., Collet P., de Coninck J. \& Dunlop F., Phys. Rev. Lett. 65, 195 (1990); J. Stat. Phys. 61 (1990) 509.

[7] Chopard B., J. Phys. A 24 (1991) L345

[8] Abraham D.B., Heinio J. \& Kaski K., J. Phys. A 24 (1991) L309; Phys. Rev. B 45 (1992) 4409

[9] Yang J.X., Koplik J. \& Banavar J.R., Phys. Rev. Lett. 67 (1991) 3539; Phys. Rev. A 46 (1992) 7738

[10] Nieminen J., Abraham D.B., Karttunen M. \& Kaski K., Phys. Rev. Lett. 69 (1992) 124.

[11] Lukkarinen A., Kaski K. \& Abraham D.B., Phys. Rev. E. (1995).

[12] Albrecht U., Otto A. \& Leiderer P., Phys. Rev. Lett. 68 (1992) 3192; Nieminen J.A. \& Ala-Nissila T., Europhys. Lett. 25 (1994) 593; idem, Phys. Rev. E 49 (1994) 4228.

\title{
A TRANSFERABLE TIGHT-BINDING POTENTIAL FOR TRANSITION METAL SILICIDES
}

\section{The Intriguing Case of $\mathrm{FeSi}_{2}$}

\author{
Leo Miglio \\ Physics Department, University of Milan
}

Semi-empirical methods for electronic structure calculations which combine flexibility and limited computational requirements allow for a reliable description of the link between structural features and the physical properties of technologically relevant complex materials.

A renaissance of the well-established tightbinding method for the semi-empirical calculation of electronic states in solids has taken place in the last few years owing to its ability to provide reliable interatomic potentials for total energy and molecular dynamics calculations in semiconductors and carbon structures [1]. The method has now been extended to cover transition-metal silicides, an important class of materials, both scientifically and technologically.

Metallic silicides play an increasingly important role in the fabrication of microelectronic devices owing to their excellent compatibility with technologies for manufacturing integrated circuits based on silicon Refractory disilicides such as $\mathrm{WSi}_{2}$ and

Leo Miglio has been an Associate Professor in the Physics Department, University of Milan, via Celoria, 16, I-20133 Milan, since 1993. He studied at the University of Pavia and after graduating in 1980 from the ICTP, Trieste, took up a postdoc position in the University of Milan before becoming an Assistant Professor in 1983. He has held visiting positions at the Max-Planck Institut für Strömungsforschung (Göttingen), the Instituto de Ciencias de $\mathrm{Ma}$ teriales (Madrid) and Florida State University (Tallahassee, USA).
$\mathrm{TiSi}_{2}$ are used at present, but their poor crystallographic matching with silicon generates defect-rich interfaces. On the other hand, near-noble disilicides such as $\mathrm{NiSi}_{2}$ and $\mathrm{CoSi}_{2}$ with the simple fluorite $\left(\mathrm{CaF}_{2}\right)$ structure display very sharp interfaces with silicon, to the extent that epitaxial $\mathrm{CoSi}_{2}$ has been experimented with for transistors of the permeable-basis type [2]. Attention of a different sort has recently been devoted to another member of the same family of compounds - namely $\mathrm{FeSi}_{2}$ - because it exists as a semiconducting ( $\beta$-) phase at room temperature. This phase has a distorted fluorite structure with a large number (48) of atoms in the (orthorhombic) unit cell. Being very complex, it can only be dealt with using semi-empirical methods and thus represents a meaningful example of the predictive power of tight-binding potentials.

The possibility of using $\beta$ - $\mathrm{FeSi}_{2}$ for optoelectronic devices has been explored. However, this application is partially frustrated by light absorption measurements and firstprinciples calculations [3] showing that an indirect band gap as large as $0.78 \mathrm{eV}$ is present, a value which is slightly smaller than the direct gap at the Brillouin zone centre $(0.85 \mathrm{eV})$. Secondly, owing to the difference 
Fig. 1-Schematic phase diagram for iron-silicide layers grown epitaxially on Si (111), taken from [4]. Temperatures $T$ and layer thicknesses $d$ should be understood as being approximate values owing to small differences in the growth conditions using molecular-beam epitaxy. We note that different stoichiometries and a large variety of phases are present, some of which do not appear in the bulk phase

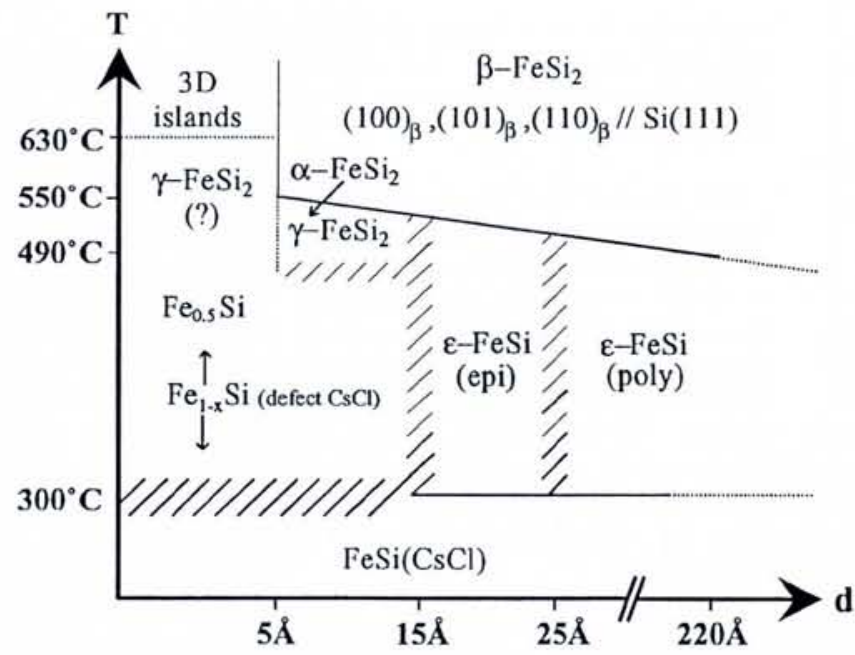

diagram, notably $\gamma$ - $\mathrm{FeSi}_{2}$, (defect $\mathrm{CsC1}$ ) $\mathrm{Fe}_{0.5} \mathrm{Si}$ and (CsCl) $\mathrm{FeSi}$, since they are stabilized by the Si (111) substrate (pseudomorphic phases). Semiconducting $\beta$-FeSi $i_{2}$ is the stable phase at room temperature and $\alpha-\mathrm{FeSi}_{2}$ is found in an epitaxial form at temperatures well below the temperature range for bulk stability.

in the crystal symmetry between the orthorombic cell and the (111) surface of silicon, it is also difficult to obtain macroscopic epitaxial samples of $\mathrm{FeSi}_{2}$ with a good crystal quality. Nonetheless, interest in fundamental aspects of the semiconducting properties of $\beta-\mathrm{FeSi}_{2}$ remains so some effort has been devoted to investigating related phases which could improve understanding. In particular, the fluorite form of the $\gamma-\mathrm{FeSi}_{2}$ phase and the defective $\mathrm{CsCl}$ structure of $\mathrm{Fe}_{0.5} \mathrm{Si}$ (where the iron sites are randomly occupied at the $50 \%$ level) have been deposited successfully by Hans von Kaenel and coworkers [4] at the ETH in Zurich using molecular-beam epitaxy. These metallic materials do not exist in the bulk, but are stabilized by a very good matching with silicon substrate, so that epitaxial films can be grown up to tens of Ângströms in thickness. At larger thicknesses, depending on temperature, a transition to the stable $\beta$-phase occurs, together with the unexpected appearance of the tetragonal $\alpha$-phase (see Fig. 1), the high-temperature bulk phase which has recently been deposited by various epitaxial techniques at temperatures well below its stability range in the bulk.

The intriguing phenomenon involving the epitaxial growth of a crystalline phase which does not appear in the bulk phase diagram is called pseudomorphism. It highlights the possibility of using epitaxy to explore structural phase transitions induced by changing neither the pressure nor the temperature [4]. Transition metal silicides are particularly interesting from this point of view, since their mainly metallic character allows for an easy transformation from one phase to another owing to the absence of high-energy barriers to atomic motion. A suitable annealing of the film, together with the substrate, even allows a transition from one stoichiometry to

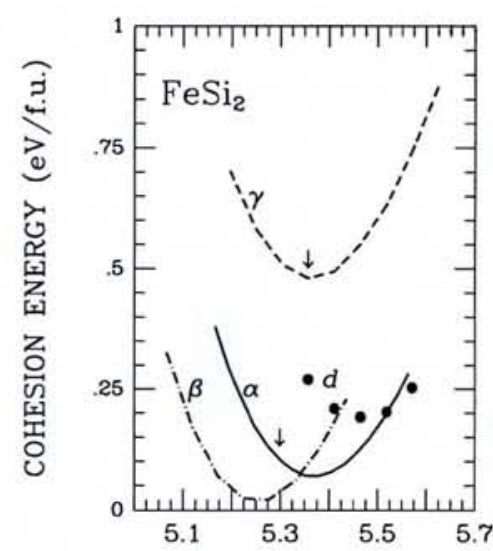

LATTICE CONSTANT $(\AA)$

Fig. 2 - The total energy curves for the various phases of $\mathrm{FeSi}_{2}$, as calculated for the bulk configuration using the semi-empirical tight-binding method. The lowest curve corresponds to the stable $\beta$-phase. At higher energies one finds the pseudomorphic phases, namely high-temperature $\alpha$ (tetragonal), $\mathrm{CsCl}$ defective and $\gamma$ (fluorite). The hierarchy in stability agrees well with the experimental data for epitaxial samples (Fig. 1), except for the high stability of the $\gamma$ (fluorite)-phase observed at very low coverages which should be ascribed to interface effects.

another (see Fig. 1). It must be appreciated, however, that the distinctive $p$-d bonding of silicides still preserves a fair amount of directional, covalent character. This probably explains why semiconducting gaps appear in the band structure depending on the atomic coordination, as is the case for $\beta-\mathrm{FeSi}_{2}$, and why a localized-orbitals scheme such as tight binding should offer an appropriate description.

\section{Semi-Empirical Schemes}

By considering the relationship between phase stability and film thickness, as described in Fig. 1, it is clear that the interface with the substrate plays a very important role in the epitaxial phase diagram of iron silicides. It is therefore very useful to have an accurate method for calculating the internal energy of structurally different and complex phases which takes account of the biaxial strain imposed by the silicon substrate in thin-film conditions. Unfortunately, since integration over the entire Brillouin zone of metallic systems demands considerable computing power, calculating the internal energy is presently beyond the capabilities of first-principles techniques. These techniques are limited to estimating the electronic density of states (DOS) and the total energy of the most simple phases in the bulk. On the other hand, fully empirical interatomic potentials do not provide electronic information, and fitting several parameters defining the total energy is difficult because very few experimental data are available, even for the bulk properties of silicides. What is needed is a semi-empirical scheme which can be fitted to the first-principles results in simple test cases and then used to extrapolate them to more realistic situations to providie electronic features, cohesion energies and elastic or vibrational informa- tion. This can be accomplished in the framework of the well-established tight-binding scheme for the calculation of the electronic states in the solids - an approach that has been applied successfully to $\mathrm{FeSi}_{2}$.

Since the late-1970s, tight-binding electronic bands have been satisfactorily included in total-energy calculations of the surface relaxation of polar semiconductors (s-p bonding) and transition metals ( $d$ - $d$ bonding) [5]. This approach can be extended to bulk transition metal silicides ( $\mathrm{p}-\mathrm{d}$ bonding), where one also requires that the parameters be transferable from one phase to another in order to obtain a coherent representation of phase diagrams. The tight-binding approach is based on the partition of the total energy $E$ of the solid into a band structure term $E_{\mathrm{bs}}$, given by the summation in reciprocal space of occupied tight-binding states $\varepsilon_{n, k}$ and a repulsive contribution $E_{\text {rep }}$ generated by a summation over the relevant neighbours $j$ of a short-range, two-body potential $f\left(\mathrm{r}_{i j}\right)$. Thus we have

$$
E=E_{\mathrm{bs}}+E_{\mathrm{rep}}=\sum_{n, k} \varepsilon_{n, k}+\sum_{i<j} \phi\left(r_{\mathrm{ij}}\right) .
$$

$E_{\mathrm{bs}}$ is negative and represents the covalent attraction provided by filling the valence electronic states which are generated by diagonalizing the Hamiltonian matrix in the localized orbitals representation. The latter includes $s$ and $p$ orbitals for silicon and $s, p$ and $d$ orbitals for the transition metal, with non-zero interactions up to the third nearestneighbours ( $\mathrm{Si}-\mathrm{Fe}, \mathrm{Si}-\mathrm{Si}$ and $\mathrm{Fe}-\mathrm{Fe}$ ) for a precise description of the DOS near the Fermi level. One then obtains the dependence of the tight-binding Hamiltonian (and of $E_{\mathrm{bs}}$ in turn) on the atomic positions by scaling its parametrized elements with distance and angle following the universal rules formulated by J.C. Slater and W.A. Harrison. 
$E_{\text {rep }}$, on the other hand, is positive and phenomenologically represents the quantum mechanical repulsion between occupied orbitals. It is obtained by fitting $f\left(r_{i j}\right)$ to the equilibrium lattice parameters and bulk moduli of a few simple phases, using equilibrium and stability conditions. The parameters entering this two-body potential (and the parametrized Hamiltonian) remain limited in number, and in the case of iron silicides one obtains very satisfactory comparisons with predictions of the first-principles calculations for all the phases simply by fitting the electronic structure, the lattice parameter and the bulk modulus of $\gamma-\mathrm{FeSi}_{2}$ and $(\mathrm{CsCl}) \mathrm{FeSi}$.

\section{Some Interesting Results}

Fig. 2 gives the calculated cohesion energy curves as a function of lattice parameter for the four $\mathrm{FeSi}_{2}$ phases. The $\beta$-phase is correctly predicted to be at the lowest energy for a zero-temperature situation. The hierarchy in stability between the high-temperature $\alpha$-phase and the defective $\mathrm{CsCl}$ phase agrees with experimental data showing that by annealing the latter one obtains the former, and eventually the $\beta$-phase. The high stability of the $\gamma$-fluorite phase in ultrathin films (see Fig. 1) is surprising since it lies at much higher energy in the phase diagram for the bulk. This can be understood by considering the stabilizing effect provided by the interface bonding to the substrate, which is likely to be very strong for the fluorite structure since its crystallography is closer to that of silicon than for the other phases.

It is interesting to monitor the origin and the nature of the band gap for the transformation from metallic $\gamma$-FeSi ${ }_{2}$ to semiconducting $\beta$-FeSi. Fig. 3a gives the sequence of several DOS, corresponding to one supercell of 48 atoms, where the progressive distortion of the atomic positions on moving from $\gamma$ to $\beta$ is represented by a unique configurational parameter $\varepsilon$ ranging from 0 to 1 , respectively. One notes that a band gap is generated slightly beyond the mid-point owing to a shift to higher energies of a nonbonding p-d peak. The total energy as a function of $\varepsilon$ is plotted in Fig. 3b: an energy barrier is not found on varying the distortion so the high stability of epitaxial $\gamma-\mathrm{FeSi}_{2}$ at very low coverages should be ascribed to interfacial bonding, which is not included in the computer calculation, and not to kinetic effects.

It is worthwhile to point out that the deformation of the lowest conduction band during the structural distortion is such that the smallest band gap turns out to be the direct one at the Brillouin zone centre for all structures with $\varepsilon<0.9$ (see Fig. 3c), and that the situation is reversed only when the actual configuration of the atomic positions for the $\beta$-phase is approached at $\varepsilon=1$. So a strong electron-phonon coupling should be present
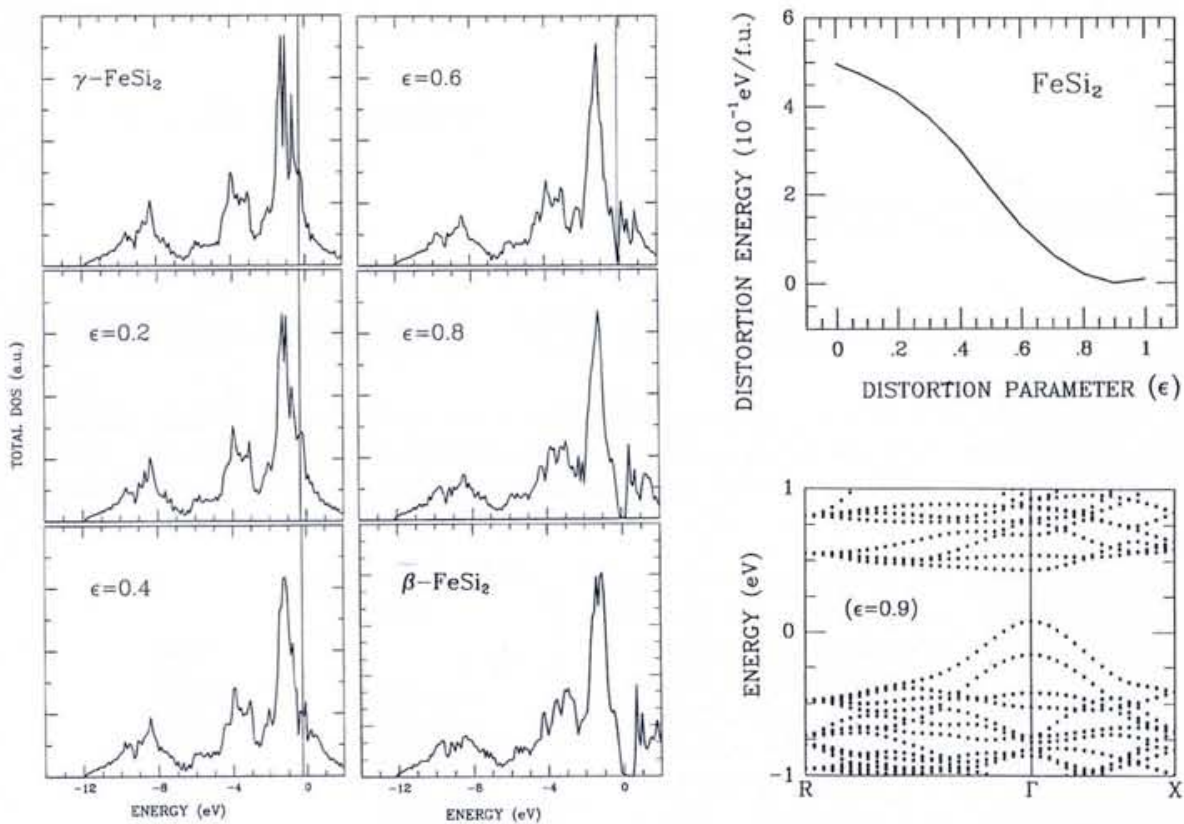

Fig. 3 - The $\beta$-phase is generated by moderate distortion of $\gamma$ - $\mathrm{FeSi}_{2}$ with a fluorite structure. The progressive changes in the electronic structure and in the total energy are illustrated as a function of the configurational parameter $\varepsilon$ which represents a linear modification of the atomic positions and the cell size on moving from from one phase to the other. Density-ofstates diagram (a, left) showing the appearance of a semiconducting band gap. Distortion energy ( $b$, upper right) showing a decrease in the total energy on moving from from $\gamma$ to $\beta$, with no activation barrier in between. The relative stability of the former at low epitaxial coverages is therefore only related to interfacial contributions which are not included. Band structure (c, lower right) for the lowest energy configuration at $\varepsilon=0.9$ close to the $\varepsilon$ value for the actual $\beta$-phase. The band gap is still direct at this level of distortion, but changes to an indirect one at $\varepsilon=1.0$, indicating that a strong electron-phonon interaction is present.

in this case, and calculation of the phonon spectrum would be very interesting.

The phonon spectrum can be estimated by performing a molecular-dynamics simulation of atomic motions at finite temperature and then evaluating the time-frequency Fourier transform of the velocity-velocity correlation function with time for each atom. The evolution with time of the atomic vibrations around the equilibrium positions is obtained using Newton's law, where the position-dependent forces are derived from the tight-binding potential [1].

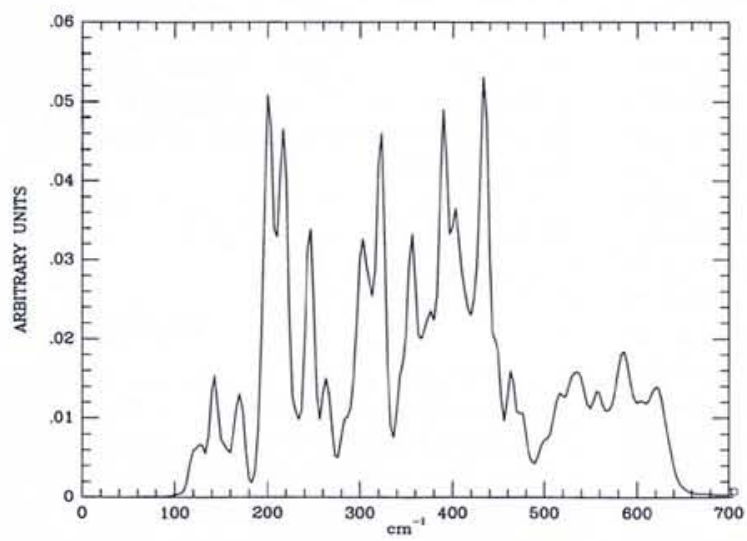

Fig. 4 - The phonon density of states at the Brillouin zone centre for $\beta$-FeSi, calculated using molecular dynamics simulations with a tight-binding interatomic potential. Despite the complexity of the spectrum, there is good agreement with optical measurements. This confirms that dynamic features can be obtained as part of a coherent framework based on using the total-energy potential which was employed to calculate electronic structures and cohesion energies.
Fig. 4 gives the phonon-density spectrum at the Brillouin zone centre for $\beta-\mathrm{FeSi}_{2}$. Many peaks are resolved owing to the preof a large number (48) of atoms in the

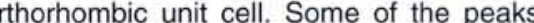
infrared active while others are Raman active, in quantitative agreement with expe-

In conclusion the examples described illustrate that calculations based on semiempirical interatomic potentials are able to provide a coherent picture of structural, electronic, vibrational, and elastic features of complex phases. So they offer materials science the possibility to systematically screen potentially interesting systems prior to carrying out costly experiments and extensive first-principles calculations.

[1] Wang C.Z., Ho K.M. \& Chen C.T., Comp. Mat. Sci. 2 (1994) 93; Molteni C., Colombo L. \& Miglio L., Europhys. Lett. 24 (1993) 659.

[2] Von Kaenel H., Mater. Sci. Rep. 6 (1991) 53.

[3] Christensen N.E., Phys. Rev. B 42 (1990) 7148; Eisebitt S. et al., Phys. Rev. B 50 (1994) 18330.

[4] Von Kaenel H., Onda N. \& Miglio L.: "Heteroepitaxy" in Science \& Technolgy of Thin Films (World Scientific) 1995. [5] Chadi D.J., Phys. Rev. B 19 (1979) 2974 